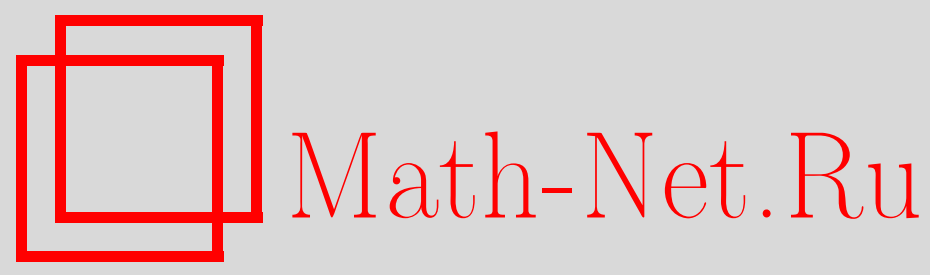

Б. Н. Хабибуллин, О росте целых функций экспоненциального типа с нулями вболизи прямой, Матем. заметки, 2001, том 70, выпуск 4, 621-635

DOI: https://doi.org/10.4213/mzm774

Использование Общероссийского математического портала Math-Net.Ru подразумевает, что вы прочитали и согласны с пользовательским соглашением http://www . mathnet.ru/rus/agreement

Параметры загрузки:

IP: 54.224 .187 .69

26 апреля 2023 г., 14:06:31

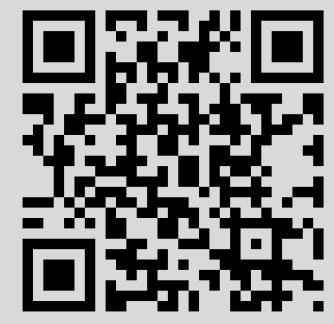




\section{О РОСТЕ ЦЕЛЫХ ФУНКЦИЙ ЭКСПОНЕНЦИАЛЬНОГО ТИПА С НУЛЯМИ ВБЛИЗИ ПРЯМОЙ}

\section{Б.Н. Хабибуллин}

Пусть $\Lambda=\left\{\lambda_{n}\right\}-$ последовательность комплексных чисел, $\lambda_{n} \rightarrow \infty$, когда $n \rightarrow+\infty$. При условии близости последовательности $\Lambda$ к мнимой оси мы получаем верхние оценки индикатора ненулевой целой функции экспоненциального типа минимального роста, обращающейся в нуль на $\Lambda$. Эти оценки дают условия, при которых система экспонент $\left\{\exp \left(\lambda_{n} z\right)\right\}$ не полна в неограниченной области в $\mathbb{C}$.

Библиографоия: 15 названий.

Введение. Пусть $\mu$ - мера на $\mathbb{C}$. Всюду в работе все меры предполагаются борелевскими и положительньми. Полагаем $\mu(r)=\mu(D(r))$, где $D(r)$ - замкнутьй круг на комплексной плоскости $\mathbb{C}$ с центром в нуле радиуса $r$.

Число

$$
\Delta_{\mu}=\varlimsup_{r \rightarrow+\infty} \frac{\mu(r)}{r},
$$

если оно конечно, назьваем, как обычно, верхней плотностью меры $\mu$ (при порядке 1).

Пусть $\Lambda=\left\{\lambda_{n}\right\}, n=1,2, \ldots,-$ последовательность комплексных чисел (точек), стремящаяся к бесконечности. С последовательностью $\Lambda$ ассоциируется мера, которую также обозначаем как $\Lambda$, и определена она по правилу

$$
\Lambda(G)=\sum_{\lambda_{n} \in G} 1, \quad G \subset \mathbb{C} .
$$

Таким образом, терминология и обозначения, касающиеся мер, естественньп образом переносятся на последовательности комплексных чисел $\Lambda$.

Целая функция $f$ обращается в нуль на последовательности $\Lambda$, если кратность нуля функции $f$ в каждой точке $\lambda \in \mathbb{C}$ не меньше числа точек $\lambda_{n} \in \Lambda$, равных $\lambda$. Последовательность $\Lambda$ назьваем последовательностью нулей целой функции $f$, если кратность нуля функции $f$ в каждой точке $\lambda \in \mathbb{C}$ равна числу повторений точки $\lambda$ в последовательности $\Lambda$.

Индикатор субгармонической в $\mathbb{C}$ функции $u$ конечного типа (при порядке 1 ), обозначаем, как обычно [1], через $h_{u}(\theta)$. Для индикатора субгармонической функции $\log |f(z)|$,

Работа выполнена при поддержке Российского фонда фундаментальных исследований, грант № 00-01-00770. 
где $f$ - целая функция экспоненциального типа (ЦФЭТ), наряду с обозначением $h_{\log }|f|$ используем и обозначение $h_{f}$.

Через $\mathbb{R}_{+}$(соответственно $\mathbb{R}_{-}$) обозначаем положительную (соответственно отрицательную) полуось вещественной оси без нуля. Таким образом, $i \mathbb{R}_{+}$(соответственно $i \mathbb{R}_{-}$) означает верхнюю (соответственно нижнюю) от нуля мнимую полуось.

Введем вариант произведения Вейерштрасса-Адамара [1], построенного по последовательности $\Lambda=\left\{\lambda_{n}\right\}, \lambda_{n} \neq 0$, конечной верхней плотности:

$$
W(z ; \Lambda)=\prod_{n}\left(1-\left(\frac{z}{\lambda_{n}}\right)^{2}\right) .
$$

В работе Красичкова-Терновского [2] в связи с задачей спектрального синтеза на выпуклых областях доказаны следующие результаты.

Tеорема А [2, теорема 8.3]. Пусть последовательность $\Lambda \subset i \mathbb{R}_{+}$имеет конечную верхнюю плотность. Тогда для любого $\varepsilon>0$ существует последовательность $\Lambda^{\prime} \subset i \mathbb{R}_{+}$конечной верхней плотности такая, что индикатор ЦФЭТ $W(z)=W(z ; \Lambda) W\left(z ; \Lambda^{\prime}\right)$ удовлетворяет условию $h_{W}( \pm \pi / 2) \leqslant \varepsilon$.

Эта теорема сьграла также важную роль при доказательствах обобщения теоремы Рубеля-Мальявена [3] о росте ЦФЭТ с предписанњыми нулями вдоль прямой и критерия полноты системы экспонент в неограниченной вьпуклой области в наших рабо$\operatorname{Tax}[4]-[6]$.

Теорема В [2, теорема 8.5]. Пусть $\Lambda_{\beta}$ - подпоследовательность последовательности $\Lambda=\left\{\lambda_{n}\right\}$ конечной верхней плотности, состоящая из точек $\lambda_{n}$, принадлежаших углу $|\arg z-\pi / 2| \leqslant \beta$. Для любого $\varepsilon>0$ существуют $\beta>0$, зависящее от $\varepsilon$, и последовательность $B=\left\{b_{n}\right\} \subset i \mathbb{R}_{+} c$ конечной верхней плотностью такие, что индикатор ЦФЭТ $W(z)=W\left(z ; \Lambda_{\beta}\right) W(z ; B)$ удовлетворяет условию $h_{W}( \pm \pi / 2) \leqslant \varepsilon$.

Далеевсюду под полнотой системы функций в области из Спонимается, полнота этой системы в пространстве голоморфных в этой области функций исключительно в топологии равномерной сходимости на компактах.

СлЕДСТВИЕ [2, следствие 8.7]. Если последовательность $\Lambda=\left\{\lambda_{n}\right\}$ имеет конечную верхнюю плотность и сәущается $\kappa$ верхней мнимой полуоси в том смысле, что $\arg \lambda_{n} \rightarrow \pi / 2$ при $n \rightarrow \infty$, то система $\left\{e^{ \pm \lambda_{n} z}\right\}$ не полна ни в какой открытой горизонтальной полосе положстельной иирины.

Оригинальные доказательства этих результатов потребовали в свое время достаточно сложных технических приемов и в полной мере использовали тонкие аналитические результаты объемной работы о сдвигах нулей целых функций [7] того же автора. В настоящей работе для доказательства приведенных результатов предлагается принципиально иной метод из [8], которьй позволяет их обобщить и уточнить. Доказанные ниже количественные варианты теорем А и В дают возможность учесть рост функций $W$ как вдоль мнимой, так и вдоль вещественной оси. В качестве применения мы указьваем усиление приведенного выше следствия, заменяя полосу на неограниченные, вообще говоря, невыпуклые области, сужающиеся на бесконечности к лучу, параллельному положительной полуоси. Сформулируем основные результаты работы. 
ОСНовНАЯ ТЕОРЕМА. Пусть число $\beta \in(0, \pi / 2)$ и $\mu$-мера конечной верхней плотности $\Delta_{\mu}>0$, носитель которой содержится в паре вертикальных углов

$$
\left\{z:\left|\arg z-\frac{\pi}{2}\right| \leqslant \beta\right\} \cup\left\{z:\left|\arg z+\frac{\pi}{2}\right| \leqslant \beta\right\} .
$$

Тогда для любой субгармонической функиии и $е$ с распределением масс Рисса $\mu$ найдется иелая функиия $g$ с нулями только на мнимой и вещественной осях такая, что субгармоническая функиия $u_{\mu}+\log |g|$ конечного типа при порядке 1 и для ее индикатора $h_{u_{\mu}+\log |g|}$ выполнена оценка

$$
h_{u_{\mu}+\log |g|}(\theta)<\frac{12 \pi(\pi+2) \Delta_{\mu}}{\pi / 2-\beta} \cdot(|\cos \theta| \operatorname{ctg} \beta+|\sin \theta| \operatorname{tg} \beta), \quad \theta \in[-\pi, \pi] .
$$

Если требовать выполнение только оценки

то при условии

$$
h_{u_{\mu}+\log |g|}\left( \pm \frac{\pi}{2}\right)<\frac{12 \pi(\pi+2) \Delta_{\mu}}{\pi / 2-\beta} \cdot \operatorname{tg} \beta
$$

$$
\left|\int_{1<|\zeta| \leqslant r} \operatorname{Re} \frac{1}{\zeta} d \mu(\zeta)\right|=O(1), \quad r \rightarrow+\infty,
$$

мохсно выбрать функиию $g$ с нулями только на мнимой оси.

В случае, когда в роли меры $\mu$ выступает мера $\Lambda$, ассоциированная с последовательностью комплексных точек $\Lambda$, субгармоническая функция $u_{\Lambda}$ с распределением масс Рисса $\Lambda$ представляется в виде $u_{\Lambda}=\log \left|f_{\Lambda}\right|$, где $f_{\Lambda}-$ целая функция с последовательностью нулей $\Lambda[9$, лемма 10.10], и из основной теоремы сразу вытекает

СлЕДСТВИЕ 1. Пусть число $\beta \in(0, \pi / 2)$ u $\Lambda=\left\{\lambda_{n}\right\}$ - последовательность конечной верхней плотности $\Delta_{\Lambda}>0$, которая, начиная с некоторого номера $n$, содержится в паре вертикальных углов (0.2). Тогда для любой целой функиии $f_{\Lambda}$ с последовательностью нулей $\Lambda$ существует иелая функиия $g$ с нулями только на вещественной и мнимой осях такая, что произведение $f=f_{\Lambda} g-$ ЦФЭТ идля индикатора $h_{f}$ функиии $f$ выполнена оценка

$$
h_{f}(\theta)<\frac{12 \pi(\pi+2) \Delta_{\Lambda}}{\pi / 2-\beta} \cdot(|\cos \theta| \operatorname{ctg} \beta+|\sin \theta| \operatorname{tg} \beta), \quad \theta \in[-\pi, \pi] .
$$

Если при дополнительном условии

$$
\left|\sum_{1<\left|\lambda_{n}\right| \leqslant r} \operatorname{Re} \frac{1}{\lambda_{n}}\right|=O(1), \quad r \rightarrow+\infty,
$$

требовать выполнение лишь оченки

$$
h_{f}\left( \pm \frac{\pi}{2}\right)<\frac{12 \pi(\pi+2) \Delta_{\Lambda}}{\pi / 2-\beta} \cdot \operatorname{tg} \beta,
$$

то мохсно выбрать функиию $g$ с нулями только на мнимой оси.

Это следствие можно рассматривать как развитие теоремы В, которая содержится в оценке (0.3) при достаточно малом $\beta$, правда без учета специального вида (0.1) функции $W$.

Для последовательности комплексных чисел $\Lambda \subset \mathbb{C}$ определим систему кратных экспонент

$$
\operatorname{Exp} \Lambda=\left\{z^{k-1} \exp (\lambda z): \lambda \in \Lambda, 1 \leqslant k \leqslant k(\lambda)\right\},
$$

где $k(\lambda)$ - число повторений точки $\lambda$ в последовательности $\Lambda$. 
СлЕДСТВИЕ 2. При тех же предположениях, что и в следствии 1, система $\operatorname{Exp} \Lambda$ не полна ни в какой области, содержащей открытый прямоугольник со сторонами, параллельными осям координат, проекиии которых на мнимую и вещественную ось равны соответственно

$$
\frac{24 \pi(\pi+2) \Delta_{\Lambda}}{\pi / 2-\beta} \cdot \operatorname{tg} \beta \quad u \quad \frac{24 \pi(\pi+2) \Delta_{\Lambda}}{\pi / 2-\beta} \cdot \operatorname{ctg} \beta .
$$

Говорим, что последовательность комплексных точек $\Lambda=\left\{\lambda_{n}\right\}$ сәущается $\kappa$ мнимой оси, если

$$
\lim _{n \rightarrow+\infty} \frac{\operatorname{Re} \lambda_{n}}{\left|\lambda_{n}\right|}=0 .
$$

Как развитие теоремы А может рассматриваться

ТЕорема 1. Пусть последовательность $\Lambda$ конечной верхней плотности $\Delta_{\Lambda}$ сгущается кмнимой оси и $f_{\Lambda}$ - целая функция с последовательностью нулей $\Lambda$. Тогда для любого достаточно малого $\varepsilon>0$ мохно подобрать иелую функиию $g c$ нулями только на вещественной и мнимой осях такую, что произведение $f=f_{\Lambda} g$ - ЦФЭТ и для индикатора $h_{f}$ функиии $f$ одновременно выполнены оценки

$$
h_{f}\left( \pm \frac{\pi}{2}\right)<\varepsilon \quad u \quad \max \left\{h_{f}(0), h_{f}(\pi)\right\} \leqslant \frac{61000 \Delta_{\Lambda}^{2}}{\varepsilon} .
$$

Если требовать выполнение лищь первой оценки в (0.10), то при условии (0.7) можсно выбрать функиию $g$ с нулями только на мнимой оси.

Конечно же, абсолютные постоянные $12 \pi(\pi+2), 24 \pi(\pi+2)$ и 61000 в оценках $(0.3)$, $(0.4),(0.6)$ и (0.8)-(0.10) далеки от наилучших возможных, что можно проследить и по доказательству сформулированных результатов. Появление знаменателя $\pi / 2-\beta$ в этих оценках, по всей видимости, обусловлено методом доказательства, и при $\beta$, близких к нулю, его влияние несущественно. В то же время, используя результаты работы [6], можно построить примеры последовательностей $\Lambda$, показывающие, что обратно пропорциональная зависимость в (0.6) между возможным минимальным ростом ЦФЭТ $f$, обращающейся в нуль на $\Lambda$, вдоль вещественной оси и вдоль мнимой оси при $\beta$, близких к нулю, существенна.

Следующее утверждение содержит в себе сформулированное вьше следствие [2, следствие 8.7].

СлЕДСТВИЕ 3. Пусть $G$ - область в $\mathbb{C}$, которая содержит луч $l$, параллельный вещественной оси, и вместе $c$ каждой точкой $x_{0}+i y_{0} \in l$ содержит все точ$\kappa и$ вертикального интервала $\left\{x_{0}+i y \in \mathbb{C}:-y_{-}\left(x_{0}\right)<y-y_{0}<y_{+}\left(x_{0}\right)\right\}$. Если $|t| y_{-}(t) \rightarrow+\infty$ или $|t| y_{+}(t) \rightarrow+\infty$ при $t \rightarrow+\infty$ или при $t \rightarrow-\infty$ и последовательность $\Lambda$ конечной верхней плотности сгущается $к$ мнимой оси, то система $\operatorname{Exp} \Lambda$ не полна в области $G$.

Очевидно, всякая горизонтальная полоса есть область вида, указанного в следствии 3, с постоянными функциями $y_{-}(t)$ и $y_{+}(t)$, и при этом области $G$ из следствия 3 не исчерпываются (полу-)полосами, а могут быть невыпуклыми и сужающимися к лучу $l$. 
1. Использование функций Йенсена. Мера $m$ с компактным носителем в $\mathbb{C}$ на-

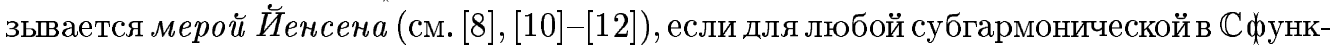
ции $u$ вьполнено неравенство $u(0) \leqslant \int u d m$.

Пусть $m$-мера Й Иенсна. Потенииалом меры $m$ (см. [8]-[10]) будем назьвать функцию

$$
V_{m}(\zeta)=\int \log |z-\zeta| d m(z)-\log |\zeta|
$$

Субгармоническую в $\mathbb{C} \backslash\{0\}$ функцию $V$ называем функиией Йенсена [8], если $V(\zeta) \geqslant 0$ при $\zeta \in \mathbb{C} \backslash\{0\}$, существует число $R>0$ такое, что $V(\zeta) \equiv 0$ при $|\zeta| \geqslant R$, и справедливо соотношение

$$
\varlimsup_{\zeta \rightarrow 0} \frac{V(\zeta)}{-\log |\zeta|} \leqslant 1
$$

ПРЕДЛОЖЕНИЕ 1.1 (ср. [8, теорема 3]). Пусть $\mu$-мера в $\mathbb{C}$, сосредоточенная вне некоторой окрестности нуля, и $p_{\nu}$ - непрерывная субгармоническая в $\mathbb{C}$ функция с распределением масс Рисса $\nu$. Если существует постоянная $C$ такая, что для всех функиий Йенсена $V$ выполнено неравенство

$$
\int V d \mu \leqslant \int V d \nu+C
$$

то для любой субгармонической функции ин с распределением масс Рисса $\mu$ найдется целая функиия $g \not \equiv 0$, для которой выполнено соотношение

$$
u_{\mu}(z)+\log |g(z)| \leqslant \sup _{|z-\zeta| \leqslant 1} p_{\nu}(\zeta)+4 \log (2+|z|), \quad z \in \mathbb{C}
$$

ДокАЗАТЕЛЬСтво. Условие (1.1) можно заменить на условие (см. [8, предложение 4.1$])$ : сушествует постоянная $C$ такая, что

$$
\int V_{m} d \mu \leqslant \int V_{m} d \nu+C
$$

для всех мер Йенсена $m$. Последнее условие можно переписать в виде (см. [8, предложение 1.4])

$$
\int u_{\mu} d m \leqslant \int p_{\nu} d m+C-p_{\nu}(0)+u_{\mu}(0)
$$

для всех мер Йенсена $m$. Отсюда согласно частному случаю [12, теорема 1] для некоторой целой функции $g \not \equiv 0$ имеем (1.1).

Предложение 1.1 доказано.

Если в предложении 1.1 взять субгармоническую функцию $p_{\nu}(x+i y)=\pi a|x|+\pi b|y|$, $x+i y \in \mathbb{C}$, где $a$ и $b$ - неотрицательные постоянные, то, как нетрудно показать, распределение масс Рисса $\nu$ действует по правилу

$$
\int V d \nu=\int_{-\infty}^{+\infty} V(x) b d x+\int_{-\infty}^{+\infty} V(i y) a d y
$$

и предложение 1.1 переходит в 
ПРЕДЛОЖЕНИЕ 1.2. Пусть $\mu$ - мера в $\mathbb{C}$, сосредоточенная вне некоторой окрестности нуля, а и $b$ - неотрицательные постоянные. Если существует постоянная $C$ такая, что

$$
\int V d \mu \leqslant b \int_{-\infty}^{+\infty} V(x) d x+a \int_{-\infty}^{+\infty} V(i y) d y+C
$$

для всех функиий Йенсена, то для любой субгармонической функчии и делением масс Рисса $\mu$ найдется целая функиия $g \not \equiv 0$, для которой выполнено соотношение

$$
u_{\mu}(x+i y)+\log |g(x+i y)| \leqslant \pi a|x|+\pi b|y|+4 \log (2+|z|), \quad z=x+i y \in \mathbb{C} .
$$

2. Переход к целым функциям с нулями на лучах. Имея в виду применения утверждений этого пункта и в других работах, приводим их для функций произвольного порядка $\rho \in \mathbb{R}_{+}$.

Замкнутое множество лучей $S$ на комплексной плоскости с началом в нуле называем системой лучей и одновременно рассматриваем $S$ и как множество точек на $\mathbb{C}$. Связные компоненты множества $\mathbb{C} \backslash S$ назьваем углами, дополнительными $\kappa S$ [13].

Систему лучей $S$ называем $\rho$-допустимой, если раствор любого угла, дополнительного к $S$, меньше, чем $\pi / \rho[13$, определение 1.4$]$.

Пусть $s$ - непустое замкнутое $2 \pi$-периодическое подмножество в $\mathbb{R}$, т.е. характеристическая функция подмножества $s$ имеет период $2 \pi$. Такое подмножество $s$ назьваем множеством направлений, а связные компоненты его дополнения в $\mathbb{R}$ назьваем интервалами, дополнительными к s.

Множество направлений $s$ назьваем $\rho$-допустимы.м, если длина любого интервала, дополнительного к $s$, меньше, чем $\pi / \rho$.

Очевидно, система лучей $S \rho$-допустима тогда и только тогда, когда множество значений аргументов точек из $S \rho$-допустимо.

Пусть $u$-субгармоническая функция в $\mathbb{C}$. Выметанием функиии и на систему лучей $S$ называем субгармоническую функцию $u_{S}^{b}$, гармоническую вне $S$ и такую, что $u(z)=u_{S}^{b}(z)$ для всех точек $z \in S[13$, определение 2.1].

Пусть $h$ - $\rho$-тригонометрически вьпуклая $2 \pi$-периодическая функция на $\mathbb{R}$. Bb.метанием функиии $h$ на множество направлений $s$ назьваем $\rho$-тригонометрически вьпуклую $2 \pi$-периодическую функцию $h_{s}^{b}, \rho$-тригонометрическую вне $s$ и такую, что $h(\theta)=$ $h_{s}^{b}(\theta)$ для всех точек $\theta \in s$.

ПРЕДЛОЖЕНИЕ 2.1. Пусть $S$ - -допустимая система лучей и множество направлений

$$
s=\left\{\theta: e^{i \theta} \in S\right\} .
$$

Если и - субгармоническая функиия конечного типа при порядке $\rho$ с индикатором $h$ (при порядке $\rho), u \not \equiv-\infty$, то существует выметание $u_{S}^{b}$ функиии и, которое является субгармонической функиией конечного типа при порядке $\rho$ с индикатором (при порядке $\rho)$, не превышающим выметание $h_{s}^{b}$ индикатора $h$. При этих требованиях выметания $u_{S}^{b}$ и $h_{s}^{b}$ единственны.

Кроме того, если дано представление функиии и $=u_{1}+u_{2}$ в виде суммы двух субгармонических функиий $u_{1}$ и $u_{2}$ с распределениями масс Рисса соответственно 
$\mu_{1} u \mu_{2}$, то выметание $u_{S}^{b}=u_{1}^{b}+u_{2}^{b}$, әде $u_{1}^{b} u u_{2}^{b}-$ выметания функций $u_{1}$ и $u_{2}$ на $S$ с распределениями масс Рисса $\mu_{1}^{b}$ и $\mu_{2}^{b}$ соответственно, сосредоточенными на $S$ и конечной верхней плотности при порядке $\rho$, причем $\mu_{1}^{b}+\mu_{2}^{b}=\mu_{S}^{b}-$ распределение масс Рисса субгармонической функиии $u_{S}^{b}, a u_{1} \leqslant u_{1}^{b} u u_{2} \leqslant u_{2}^{b}$ всюдуна $\mathbb{C}$.

Это предложение доказано в [13, леммы 2.3 и 2.2], кроме единственности $u_{S}^{b}$ и последнего утверждения о функции $u$, представленной в виде суммы двух функций. Единственность легко следует из принципа Фрагмена-Линделёфа для угла [14, теорема 1.7.4]. Последнее утверждение содержится в явной конструкции выметаний $u_{S}^{b}$ и $\mu_{S}^{b}$ из $[13, \S 1$, $\S 2]$, где два последних неравенства вновь вытекают из принципа Фрагмена-Линделёфа, примененного ко всем углам, дополнительным к $S$.

ПРЕДЛОЖЕНИЕ 2.2. Пусть $S$ - - -допустимая система из конечного числа лучей. Если и и - субгармонические функиии и сумма $u+v-$ субгармоническая функиия конечного типа при порядке $\rho$ с индикатором $h$ (при порядке $\rho)$, то найдется иелая функиия $g$ с нулями только на $S$ такая, что индикатор субгармонической функиии $u+\log |g|$ не превышает выметания $h_{s}^{b}$ функиии $h$ на множество направлений $s$, определенное в (2.1).

ДокАЗАТЕЛЬСТВо. Построим выметание субгармонической функции $u+v$ на систему лучей $S$, которое по предложению 2.1 есть субгармоническая функция конечного типа при порядке $\rho$ с индикатором (при порядке $\rho$ ) не вьше $h_{s}^{b}$ и представляется в виде $u_{S}^{b}+v_{S}^{b}$, где $u \leqslant u_{S}^{b}$ и $v \leqslant v_{S}^{b}$ на $\mathbb{C}$. Из предпоследнего неравенства следует, что функция $u+v_{S}^{b}$ конечного типа при порядке $\rho$ с индикатором (при порядке $\rho$ ), не превышающим $h_{s}^{b}$.

При этом распределение масс Рисса функции $v_{S}^{b}$ имеет конечную верхнюю плотность при порядке $\rho$, так как функция $u_{S}^{b}+v_{S}^{b}$ конечного типа при порядке $\rho[14$, гл. I, $\S 3$, п. 1$]$, и по определению выметания это распределение масс сосредоточено на конечной системе лучей $S$. Следовательно, справедливо представление $v_{S}^{b}=w+H$, где $w$ - субгармоническая функция порядка не вьше $\rho$ с распределением масс Рисса на $S$, а $H$ - гармоническая функция [14, теорема 1.3.1]. Отсюда субгармоническая функция $u+H=\left(u+v_{S}^{b}\right)-w$ также функция порядка не вьше $\rho$ как разность субгармонических функций порядка не выше $\rho[14$, лемма 1.3.2].

Теперь воспользуемся частным случаем одного результата, вытекающего из ККК (Kjellberg-Kennedy-Katifi) аппроксимационного метода [9, п. 10.5].

ЛЕмма 2.1 [9, лемма 10.12]. Пусть $w$ - субгармоническая функиия с распределением масс Рисса, сосредоточенным на конечной системе лучей $S$. Тогда существует иелая функиия $q$ с нулями только на $S$ такая, что

$$
\log |q(z)|=w(z)+O\left(\log ^{+} \frac{1}{\operatorname{dist}(z, S)}\right)+O(\log |z|), \quad z \rightarrow \infty
$$

әде $\log ^{+} t=\max \{\log t, 0\} u \operatorname{dist}(z, S)-$ расстояние от точки z до множсества $S$; при этом

$$
T(r, \log |q|)=T(r, w)+O(\log r), \quad r \rightarrow+\infty,
$$

где $T(r, \log |q|)$ и $T(r, w)$ - характеристики Неванлинны функиий $\log |q|$ и $w$. 
Применяя эту лемму, найдем целую функцию $q$ с нулями только на $S$ такую, что согласно (2.2) для любого $\varepsilon>0$ выполнено соотношение

$$
\begin{aligned}
u(z)+H(z)+\log |q(z)| & \leqslant u(z)+H(z)+w(z)+O(\log |z|) \\
& \leqslant\left(h_{s}^{b}(\arg z)+\varepsilon\right)|z|^{\rho}+O(\log |z|), \quad \operatorname{dist}(z, S) \geqslant 1, \quad z \rightarrow \infty
\end{aligned}
$$

и по свойствам характеристики Неванлинны согласно (2.3)

$$
\begin{aligned}
T(r, u+H+\log |q|) & \leqslant T(r, u+H)+T(r, \log |q|) \\
& \leqslant T(r, u+H)+T(r, w)+O(\log r), \quad r \rightarrow+\infty,
\end{aligned}
$$

откуда следует, что функция $u+H+\log |q|-$ функция порядка не вьше $\rho$ ввиду известной взаимосвязи между ростом характеристики Неванлинны и максимумом субгармонической функции на окружностях с центром в нуле [15, теорема 3.19]. В силу гармоничности $H$ найдется целая функция $p$ такая, что $\operatorname{Re} p=H$. Полагаем $g=q \exp p$-целая функция с нулями только на $S$ такая, что функция $u+\log |g|$ порядка не вьше $\rho$ и ввиду (2.4) для любого $\varepsilon>0$ вьполнено соотношение

$$
u(z)+\log |g(z)| \leqslant\left(h_{s}^{b}(\arg z)+\varepsilon\right)|z|^{\rho}+O(\log |z|), \quad \operatorname{dist}(z, S) \geqslant 1, \quad z \rightarrow \infty .
$$

Избавимся в $(2.5)$ от ограничения $\operatorname{dist}(z, S) \geqslant 1$.

Пусть $l$ - луч из системы $S$. Не умаляя общности, можно считать, что $l=\mathbb{R}_{+}$. Тогда ввиду конечности $S$ согласно (2.5) для сколь угодно малых $\delta>0$ при $|\arg z|=\delta$ и $z \rightarrow \infty$ выполнено асимптотическое соотношение из (2.5). Гармонически продолжая субгармоническую функцию $K(z)=\left(h_{s}^{b}(\arg z)+\varepsilon\right)|z|^{\rho}$ внутрь угла $|\arg z|<\delta$ при достаточно малом $\delta>0$, получим непрерьвную субгармоническую функцию $K_{\delta}\left(r e^{i \theta}\right)$, которая всюду мажорирует функцию $K(z)$, внутри угла $|\theta|<\delta$ имеет вид $(A \cos \theta+B \sin \theta) r^{\rho}$ и ввиду непрерьвности $\rho$-тригонометрически выпуклых функций [1] мажорируется там функцией $\left(h_{s}^{b}(\theta)+2 \varepsilon\right) r^{\rho}$. Отсюда и из асимптотического соотношения (2.5) на лучах $|\theta|=\delta$ по принципу Фрагмена-Линделёфа в угле $|\theta|<\delta$, примененного к субгармонической в нем функции $u+\log |g|-K_{\delta}$, получаем

$$
u\left(r e^{i \theta}\right)+\log \left|g\left(r e^{i \theta}\right)\right| \leqslant\left(h_{s}^{b}(\theta)+2 \varepsilon\right) r^{\rho}+O(\log r), \quad|\theta| \leqslant \delta, \quad z \rightarrow \infty .
$$

Применяя эти рассуждения к каждому лучу из $S$, убеждаемся, что индикатор функции $u+\log |g|$ при порядке $\rho$ не превьшает $h_{s}^{b}$, и предложение 2.2 доказано.

Последовательно применяя предложение 1.2 , а затем предложение 2.2 с функцией $v=\log |g|$ и системой лучей $S=\mathbb{R} \cup i \mathbb{R}$, получаем

ПРЕДЛОЖЕНИЕ 2.3. Пусть $\mu-$ мера в $\mathbb{C}$, сосредоточенная вне некоторой окрестности нуля, а и $b$ - неотрицательные постоянные. Если существует постоянная $C$ такая, что для всех функиий Йенсена выполнено (1.3), то для любой субгармонической функиии $u_{\mu}$ с распределением масс Рисса $\mu$ найдется иелая функиия $g$ с нулями только на вещественной и мнимой осях, для которой выполнено неравенство

$$
h_{u_{\mu}+\log |g|}(\theta) \leqslant \pi a|\cos \theta|+\pi b|\sin \theta|
$$

при всех $\theta \in[-\pi, \pi]$. 
3. Вспомогательные оценки. Через $\angle(\alpha, \beta)$ обозначаем открытый угол $\{z \in \mathbb{C}$ : $\alpha<\arg z<\beta\}$. Для борелевского множества $E \subset \mathbb{C}$ через $\omega(\zeta, E ; \angle(\alpha, \beta))$ обозначаем гармоническую меру пересечения множества $E$ с гранищей угла $\angle(\alpha, \beta)$ в точке $\zeta$ относительно угла $\angle(\alpha, \beta)[15]$.

ПРЕДЛОЖЕНИЕ 3.1. Пусть $\pi / \gamma=\beta-\alpha-$ раствор угла $\angle(\alpha, \beta)$. Справедлива оценка

$$
\omega(\zeta, D(t) ; \angle(\alpha, \beta)) \leqslant \frac{2}{\pi} \cdot\left(\frac{2^{\gamma}}{2^{\gamma}-1}\right)^{2} \cdot\left(\frac{t}{|\zeta|}\right)^{\gamma}, \quad|\zeta| \geqslant 2 t>0 .
$$

ДокАЗАТЕЛьство. Гармоническая мера инвариантна относительно поворотов, поэтому, не умаляя общности, можно считать, что $\angle(\alpha, \beta)=\angle(0, \pi / \gamma)$. Далее воспользуемся редукцией к верхней полуплоскости. Полагаем

$$
\zeta^{\prime}=\zeta^{\gamma} \quad \text { и } \quad t^{\prime}=t^{\gamma},
$$

где рассматривается ветвь функции $\zeta^{\gamma}$, положительная на $\mathbb{R}_{+}$. При условии $|\zeta| \geqslant 2 t>0$ имеем

$$
t^{\prime} \leqslant \frac{1}{2 \gamma}\left|\zeta^{\prime}\right| .
$$

Из конформной инвариантности гармонической меры и представления ее через интеграл Пуассона получаем

$$
\begin{aligned}
\omega\left(\zeta, D(t) ; \angle\left(0, \frac{\pi}{\gamma}\right)\right) & =\omega\left(\zeta^{\prime}, D\left(t^{\prime}\right) ; \angle(0, \pi)\right) \\
& =\frac{1}{\pi} \int_{-t^{\prime}}^{t^{\prime}} \operatorname{Im} \frac{1}{x-\zeta^{\prime}} d x=\frac{1}{\pi} \cdot \operatorname{Im} \zeta^{\prime} \int_{-t^{\prime}}^{t^{\prime}} \frac{d x}{\left|x-\zeta^{\prime}\right|^{2}}
\end{aligned}
$$

При $|x| \leqslant t^{\prime}$, используя $(3.3)$, имеем

$$
\left|x-\zeta^{\prime}\right| \geqslant\left|\zeta^{\prime}\right|-|x| \geqslant\left|\zeta^{\prime}\right|-t^{\prime} \geqslant\left(1-\frac{1}{2^{\gamma}}\right)\left|\zeta^{\prime}\right| .
$$

Отсюда согласно (3.4) и (3.2) получаем

$$
\begin{aligned}
\omega\left(\zeta, D(t) ; \angle\left(0, \frac{\pi}{\gamma}\right)\right) & \leqslant \frac{1}{\pi} \cdot \frac{\operatorname{Im} \zeta^{\prime}}{\left|\zeta^{\prime}\right|^{2}} \cdot\left(\frac{2^{\gamma}}{2^{\gamma}-1}\right)^{2} \cdot 2 t^{\prime} \\
& =\frac{2}{\pi} \cdot \frac{\operatorname{Im} \zeta^{\gamma}}{|\zeta|^{2 \gamma}} \cdot\left(\frac{2^{\gamma}}{2^{\gamma}-1}\right)^{2} \cdot t^{\gamma} \leqslant \frac{2}{\pi} \cdot \frac{1}{|\zeta| \gamma} \cdot\left(\frac{2^{\gamma}}{2^{\gamma}-1}\right)^{2} \cdot t^{\gamma}
\end{aligned}
$$

что совпадает с оценкой (3.1).

Пусть $\mu$-мера с носителем в замкнутом угле

$$
\angle[\alpha, \beta]=\{z \in \mathbb{C}: \alpha \leqslant \arg z \leqslant \beta\} \cup\{0\} .
$$

Выметание $\mu^{b}$ меры $\mu$ из угла $\angle(\alpha, \beta)$ есть мера, определяемая на борелевских множествах $E$ по правилу [13, определение 1.3]

$$
\mu^{b}(E)=\int \omega(\zeta, E ; \angle(\alpha, \beta)) d \mu(\zeta)
$$

В частности, если мера $\mu$ конечной верхней плотности (при порядке 1) и раствор угла $\angle(\alpha, \beta)$ меньше $\pi$, то выметание $\mu^{b}$ из (3.5) существует и также конечной верхней плотности (при порядке 1) [13, теорема 1.1]. 
ПРЕДЛОЖЕНИЕ 3.2. Пусть $\mu$-мера конечной верхней плотности (при порядке 1) с носителем в угле $\angle[\pi / 2-\beta, \pi / 2+\beta]$, где $\beta \in(0, \pi / 2)$, и выполнено условие

$$
\mu(t) \leqslant \Delta \cdot t, \quad t>0 .
$$

Тогда выметание $\mu^{b}$ меры $\mu$ из угла $\angle(\pi / 2-\beta, \pi / 2+\beta)$ есть мера конечной верхней плотности (при порядке 1) такая, что

$$
\mu^{b}(t)<\frac{(\pi-2 \beta+2) \Delta}{\pi / 2-\beta} \cdot t, \quad t>0
$$

ДокАЗАТЕЛьСТво. Из (3.5) имеем представление

$$
\begin{aligned}
\mu^{b}(t)= & \int_{|\zeta| \leqslant 2 t} \omega\left(\zeta, D(t) ; \angle\left(\frac{\pi}{2}-\beta, \frac{\pi}{2}+\beta\right)\right) d \mu(\zeta) \\
& +\int_{|\zeta|>2 t} \omega\left(\zeta, D(t) ; \angle\left(\frac{\pi}{2}-\beta, \frac{\pi}{2}+\beta\right)\right) d \mu(\zeta)
\end{aligned}
$$

В первом интеграле оцениваем сверху гармоническую меру единицей, а во втором интеграле по предложению 3.1 при $\pi / \gamma=2 \beta$ и получаем

$$
\mu^{b}(t) \leqslant \mu(2 t)+\frac{2}{\pi} \cdot\left(\frac{2^{\gamma}}{2^{\gamma}-1}\right)^{2} \cdot t^{\gamma} \int_{2 t}^{+\infty} \frac{d \mu(r)}{r^{\gamma}} .
$$

Отсюда согласно (3.6), интегрируя по частям последний интеграл с учетом того, что $\gamma>1$, имеем

$$
\mu^{b}(t) \leqslant 2 \Delta \cdot t+\frac{2}{\pi} \cdot\left(\frac{2^{\gamma}}{2^{\gamma}-1}\right)^{2} \cdot t^{\gamma} \cdot \Delta \cdot \frac{\gamma}{\gamma-1}(2 t)^{1-\gamma} \leqslant 2 \Delta \cdot t\left(1+\frac{4}{\pi} \cdot \frac{\gamma}{\gamma-1}\right)
$$

Подставляя в правую часть $\gamma=\pi /(2 \beta)$, получаем

$$
\mu^{b}(t) \leqslant \Delta \cdot t \cdot \frac{\pi-2 \beta+2}{\pi / 2-\beta},
$$

и предложение 3.2 доказано.

Нам потребуются оценки ядра интеграла Пуассона для первой четверти

$$
P\left(z, \zeta ; \angle\left(0, \frac{\pi}{2}\right)\right)=\left\{\begin{array}{l}
P_{\mathbb{R}_{+}}(z, x), \quad \text { когда } \zeta=x \in \mathbb{R}_{+} \cup\{0\}, \\
P_{i \mathbb{R}_{+}}(z, i y), \text { когда } \zeta=i y \in i \mathbb{R}_{+} \cup\{0\},
\end{array}\right.
$$

где для $z=r e^{i \theta} \in \angle(0, \pi / 2)$

$$
\begin{aligned}
P_{\mathbb{R}_{+}}\left(r e^{i \theta}, x\right) & =\frac{2}{\pi} \cdot \frac{r^{2} x \sin 2 \theta}{r^{4}+x^{4}-2 r^{2} x^{2} \cos 2 \theta}, \\
P_{i \mathbb{R}_{+}}\left(r e^{i \theta}, i y\right) & =\frac{2}{\pi} \cdot \frac{r^{2} y \sin 2 \theta}{r^{4}+y^{4}+2 r^{2} y^{2} \cos 2 \theta} .
\end{aligned}
$$


ПРЕДЛОЖЕНИЕ 3.3. Для $z=r e^{i \theta} \in \angle(0, \pi / 2)$ справедливы оценки

$$
\begin{gathered}
P_{\mathbb{R}_{+}}\left(r e^{i \theta}, x\right) \leqslant \frac{1}{\pi} \cdot \begin{cases}\frac{\operatorname{ctg} \theta}{x} & n p u 0<r \leqslant 2 x, \\
\frac{32}{9} \cdot \frac{x \sin 2 \theta}{r^{2}} & n p u r \geqslant 2 x,\end{cases} \\
P_{i \mathbb{R}_{+}}\left(r e^{i \theta}, i y\right) \leqslant \frac{1}{\pi} \cdot \begin{cases}\frac{\operatorname{tg} \theta}{y} & n p u 0<r \leqslant 2 y, \\
\frac{32}{9} \cdot \frac{y \sin 2 \theta}{r^{2}} & n p u r \geqslant 2 y .\end{cases}
\end{gathered}
$$

ДокАЗАТЕЛЬСТво. Выражения из (3.9) и (3.10) легко преобразовать к виду

$$
\begin{aligned}
P_{\mathbb{R}_{+}}\left(r e^{i \theta}, x\right) & =\frac{2}{\pi} \cdot \frac{r^{2} x \sin 2 \theta}{\left(r^{2}-x^{2}\right)^{2}+4 r^{2} x^{2} \sin ^{2} \theta} \\
P_{i \mathbb{R}_{+}}\left(r e^{i \theta}, i y\right) & =\frac{2}{\pi} \cdot \frac{r^{2} y \sin 2 \theta}{\left(r^{2}-y^{2}\right)^{2}+4 r^{2} y^{2} \cos ^{2} \theta}
\end{aligned}
$$

Докажем оценку (3.11). При $0<r \leqslant 2 x$ из (3.13)

$$
P_{\mathbb{R}_{+}}\left(r e^{i \theta}, x\right) \leqslant \frac{2}{\pi} \cdot \frac{r^{2} x \sin 2 \theta}{4 r^{2} x^{2} \sin ^{2} \theta}=\frac{2}{\pi} \cdot \frac{\sin 2 \theta}{4 x \sin ^{2} \theta}=\frac{2}{\pi} \cdot \frac{2 \cos \theta}{4 x \sin \theta}
$$

и получаем оценку (3.11) для $0<r \leqslant 2 x$.

При $r \geqslant 2 x$ имеем $r^{2}-x^{2} \geqslant 3 r^{2} / 4$ и из (3.13)

$$
P_{\mathbb{R}_{+}}\left(r e^{i \theta}, x\right) \leqslant \frac{2}{\pi} \cdot \frac{r^{2} x \sin 2 \theta}{\left(r^{2}-x^{2}\right)^{2}} \leqslant \frac{2}{\pi} \cdot \frac{r^{2} x \sin 2 \theta}{\left(3 r^{2} / 4\right)^{2}}=\frac{1}{\pi} \cdot \frac{32}{9} \cdot \frac{x \sin 2 \theta}{r^{2}},
$$

что дает оценку (3.11) для $r \geqslant 2 x$.

Аналогично из (3.14) получается оценка (3.12).

ПРЕДЛОЖЕНИЕ 3.4. Пусть $\theta \in(0, \pi / 2)$, мера $\mu$ сосредоточена на луче $\{z \in \mathbb{C}:$ $\arg z=\theta\}$ и удовлетворяет неравенству (3.6). Тогда справедливы оценки

$$
\begin{gathered}
\int_{0}^{+\infty} P_{\mathbb{R}_{+}}\left(r e^{i \theta}, x\right) d \mu(r) \leqslant 3 \Delta \cdot \operatorname{ctg} \theta \\
\int_{0}^{+\infty} P_{i \mathbb{R}_{+}}\left(r e^{i \theta}, i y\right) d \mu(r) \leqslant 3 \Delta \cdot \operatorname{tg} \theta
\end{gathered}
$$

ДоКАЗАТЕЛЬСТВО. Из оценки (3.11) предложения 3.3

$$
\begin{aligned}
\int_{0}^{+\infty} P_{\mathbb{R}_{+}}\left(r e^{i \theta}, x\right) d \mu(r) & \leqslant \frac{1}{\pi}\left(\int_{0}^{2 x} \frac{\operatorname{ctg} \theta}{x} d \mu(r)+\frac{32}{9} \int_{2 x}^{+\infty} \frac{x \sin 2 \theta}{r^{2}} d \mu(r)\right) \\
& =\frac{1}{\pi}\left(\frac{\operatorname{ctg} \theta}{x} \mu(2 x)+\frac{32}{9} \cdot x \sin 2 \theta \int_{2 x}^{+\infty} \frac{d \mu(r)}{r^{2}}\right)
\end{aligned}
$$


откуда, интегрируя по частям последний интеграл, согласно (3.6) получаем

$$
\begin{aligned}
\int_{0}^{+\infty} P_{\mathbb{R}_{+}}\left(r e^{i \theta}, x\right) d \mu(r) & \leqslant \frac{1}{\pi}\left(2 \Delta \cdot \operatorname{ctg} \theta+\frac{64}{9} \cdot x \sin 2 \theta \cdot \Delta \int_{2 x}^{+\infty} \frac{d r}{r^{2}}\right) \\
& =\frac{2 \Delta}{\pi}\left(\operatorname{ctg} \theta+\frac{16}{9} \sin 2 \theta\right) .
\end{aligned}
$$

В силу неравенства $\sin 2 \theta \leqslant 2 \operatorname{ctg} \theta$ это дает (3.15).

Аналогично из оценки (3.11) предложения 3.3 согласно (3.6) получаем

$$
\int_{0}^{+\infty} P_{i \mathbb{R}_{+}}\left(r e^{i \theta}, i y\right) d \mu(r) \leqslant \frac{2 \Delta}{\pi}\left(\operatorname{tg} \theta+\frac{16}{9} \sin 2 \theta\right)
$$

откуда в силу неравенства $\sin 2 \theta \leqslant 2 \operatorname{tg} \theta$ следует (3.16).

\section{4. Доказательства основных результатов.}

ДОКАЗАТЕЛЬСТВО ОСНОВНОЙ ТЕОРЕМЫ. Пусть $\Delta>\Delta_{\mu}>0$. Можно представить меру $\mu$ в виде суммы двух мер $\mu=\mu_{r}+\mu_{\infty}$, где $\mu_{r}-$ сужение меры $\mu$ на круг $D(r)$ такое, что $\mu_{\infty}(t) \leqslant \Delta \cdot t, t>0$, и, очевидно, $\mu_{r}(\mathbb{C})<+\infty$. Поскольку логарифмический потенциал

$$
\int \log \left|1-\frac{z}{\zeta}\right| d \mu_{r}(\zeta)
$$

конечной меры $\mu_{r}$ растет не быстрее величины $O(\log |z|)$ при $z \rightarrow \infty$, то его добавка не влияет на значения индикатора субгармонической функции. Следовательно, не умаляя общности, можно считать, что для меры $\mu$ вьполнено (3.6) и она сосредоточена вне некоторой окрестности нуля.

По теореме Вейерштрасса-Адамара о представлении субгармонических на плоскости функции [15], [14, теоремы 1.3.1 и 1.3.2] справедливо представление $u_{\mu}$ в виде суммы субгармонической функции $u_{1}$ порядка не вьше 1 с распределением масс Рисса $\mu$ и некоторой гармонической функции $H=\log |\exp q|$, где $q$ - целая функция. Если требуемая в основной теореме целая функция $g$ построена для $u_{1}$, то для $u_{\mu}$ такой функцией будет $g \exp q$. Следовательно, не умаляя общности, можем считать, что $u_{\mu}-$ субгармоническая функция порядка не вьше 1.

Рассмотрим выметание $u_{\mu}^{b}$ функции $u_{\mu}$ из пары вертикальных углов (0.2). Тогда распределением масс Рисса функции $u_{\mu}^{b}$ будет выметание $\mu^{b}$ меры $\mu$ из этой пары углов, построенное по правилу (3.5) (см. предложение 2.2 и [13, основная теорема]). Мера $\mu^{b}$ сосредоточена на четырех лучах

$$
\left\{z \in \mathbb{C}: \arg z= \pm \frac{\pi}{2} \pm \beta\right\}
$$

и конечной верхней плотности при порядке 1. При этом из принципа Фрагмена-Линделёфа для пары вертикальных углов $(0.2)$ следует $u_{\mu} \leqslant u_{\mu}^{b}$ всюду на $\mathbb{C}$. Кроме того, по предложению 3.2 вьполнено (3.7) с дополнительным множителем 2 в правой части.

Пусть $\mu_{k}, k=1,2,3,4,-$ сужения меры $\mu^{b}$ на лучи из (4.1), лежашие соответственно в I, II, III и IV четвертях плоскости $\mathbb{C}$. Для каждой из них справедлива оценка (3.7) с дополнительным множителем 2 в правой части и с заменой левой части на $\mu_{k}(t)$, a сумма мер $\mu_{k}$ равна мере $\mu^{b}$. 
Пусть $V$ - функция Йенсена. Для точек $\zeta$, лежащих в I четверти на луче $\{z \in \mathbb{C}$ : $\arg z=\theta\}$ с $\theta=\pi / 2-\beta$ в силу субгармоничности $V$, условия $V \equiv 0$ вне некоторого круга $D(R)$ и всего лиш логарифмической особенности в нуле справедлива в обозначениях (3.8)-(3.10) оценка через интеграл Пуассона:

$$
\begin{aligned}
& \int_{0}^{+\infty} V\left(t e^{i \theta}\right) d \mu_{1}(t) \\
& \quad \leqslant \int_{0}^{+\infty}\left(\int_{0}^{+\infty} V(x) P_{\mathbb{R}_{+}}\left(r e^{i \theta}, x\right) d x+\int_{0}^{+\infty} V(i y) P_{i \mathbb{R}_{+}}\left(r e^{i \theta}, i y\right) d y\right) d \mu_{1}(r) .
\end{aligned}
$$

Применяя теорему Фубини о повторных интегралах и оценки (3.15) и (3.16) предложения 3.4 , с учетом оценки роста для $\mu_{1}(t)$ и неотрицательности функций Йенсена получаeM

$$
\begin{aligned}
& \int_{0}^{+\infty} V\left(t e^{i \theta}\right) d \mu_{1}(t) \\
& \quad \leqslant \frac{6(\pi-2 \beta+2) \Delta}{\pi / 2-\beta}\left(\operatorname{ctg}\left(\frac{\pi}{2}-\beta\right) \int_{0}^{+\infty} V(x) d x+\operatorname{tg}\left(\frac{\pi}{2}-\beta\right) \int_{0}^{+\infty} V(i y) d y\right) .
\end{aligned}
$$

Аналогично действуя в остальных трех четвертях и суммируя оценки по четырем лучам, имеем

$$
\int V(\zeta) d \mu^{b}(\zeta) \leqslant \frac{12(\pi-2 \beta+2) \Delta}{\pi / 2-\beta}\left(\operatorname{tg} \beta \int_{-\infty}^{+\infty} V(x) d x+\operatorname{ctg} \beta \int_{-\infty}^{+\infty} V(i y) d y\right)
$$

Отсюда по предложению 2.3 найдется целая функция $g$ с нулями только на вещественной и мнимой осях, для которой выполнено неравенство

$$
h_{u_{\mu}^{b}+\log |g|}(\theta) \leqslant \frac{12(\pi-2 \beta+2) \Delta}{\pi / 2-\beta}(\pi \operatorname{ctg} \beta \cdot|\cos \theta|+\pi \operatorname{tg} \beta \cdot|\sin \theta|)
$$

при всех $\theta \in[-\pi, \pi]$. Так как $\beta>0$ и $u_{\mu} \leqslant u_{\mu}^{b}$ всюду на $\mathbb{C}$, то, выбирая $\Delta>\Delta_{\mu}$ достаточно близко к $\Delta_{\mu}$, получаем $(0.3)$, и первая часть основной теоремы доказана.

Пусть теперь выполнено условие (0.5) и сначала $g$ - целая функция с нулями только на $\mathbb{R}$ и $i \mathbb{R}$ такая, что имеет место (0.3). Так как $u_{\mu}+\log |g|-$ функция конечного типа при порядке 1 , то мера $\mu+\Lambda$ конечной верхней плотности, где $\Lambda$ - мера, ассоциированная с последовательностью нулей функции $g$, и вьполнено условие Линделёфа

$$
\left|\int_{1<|\zeta| \leqslant r} \frac{1}{\zeta} d(\mu(\zeta)+\Lambda(\zeta))\right|=O(1), \quad r \rightarrow+\infty
$$

Пусть $\Lambda_{r}=\left\{\lambda_{n}^{\prime}\right\}$ та часть последовательности $\Lambda$, которая лежит на $\mathbb{R}, \lambda_{n}^{\prime} \neq 0$. Тогда из последнего асимптотического соотношения следует, что последовательность $\Lambda_{r}$ удовлетворяет условию Линделёфа:

$$
\left|\sum_{1<\left|\lambda_{n}^{\prime}\right| \leqslant r} \frac{1}{\lambda_{n}^{\prime}}\right|=O(1), \quad r \rightarrow+\infty
$$


Отсюда по теореме Линделёфа [1, гл. I, § 11, теорема 15] справедливо представление $g=g_{r} g_{i}$, где $g_{r}-Ц Ф Э Т$ с последовательностью только вещественных нулей $\Lambda_{r}$, представленная каноническим произведением Вейерштрасса-Адамара

$$
g_{r}(z)=\prod_{n}\left(1-\frac{z}{\lambda_{n}^{\prime}}\right) \exp \left(\frac{z}{\lambda_{n}^{\prime}}\right)
$$

a $g_{i}$ - целая функция с последовательностью нулей, целиком лежащей на мнимой оси. Отсюда $u_{\mu}(z)+\log \left|g_{i}(z)\right| \leqslant$ const $\cdot|z|-\log \left|g_{r}(z)\right|$ и в силу известных [1] оценок снизу для ЦФЭТ $g_{r}$ функция $u_{\mu}+\log \left|g_{i}\right|$ конечного типа при порядке 1. Кроме того, из представления (4.2) следует, что $\log \left|g_{r}(i y)\right| \geqslant 0, i y \in i \mathbb{R}$, и из неравенства

$$
u_{\mu}(i y)+\log \left|g_{i}(i y)\right| \leqslant u_{\mu}(i y)+\log |g(i y)|
$$

согласно (0.3) следует (0.4).

Основная теорема доказана.

Следствие 1 есть просто переформулировка основной теоремы для ассоциированной с последовательностью меры $\mu=\Lambda$. Следствие 2 прямо вытекает из следствия 1 в силу известной взаимосвязи между полнотой системы $\operatorname{Exp} \Lambda$ и теоремами единственности для ЦФЭТ в терминах индикаторной диаграммы [1].

ДОКАЗАТЕЛЬСТВО ТЕОРЕМЫ 1. Если $\Delta_{\Lambda}=0$, то по теореме Линделёфа $f(z)=$ $f_{\Lambda}(z) f_{\Lambda}(-z)$ - целая функция минимального типа при порядке 1 . По предложению 2.2 функцию $f_{\Lambda}(-z)$ можно заменить на целую функцию $g$ с нулями только на вещественной и мнимой осях, а при условии (0.7) так же, как в конще доказательства основной теоремы, на целую функцию $g$ с нулями только на вещественной и мнимой осях.

Пусть $\Delta_{\Lambda}>0$. Выберем число $\beta$ так, что $0<\beta \leqslant \pi / 4$ и

$$
\varepsilon=48(\pi+2) \Delta_{\Lambda} \cdot \operatorname{tg} \beta \leqslant \frac{12 \pi(\pi+2) \Delta_{\Lambda}}{\pi / 2-\beta} \cdot \operatorname{tg} \beta
$$

В силу сгущаемости $\Lambda$ к мнимой оси, отбрасывая конечное число точек из $\Lambda$, не умаляя общности, можем считать, что последовательность $\Lambda$ целиком лежит внутри пары вертикальных углов (0.2). По следствию 1 существует целая функция $g$ с нулями только на вещественной и мнимой осях такая, что для $f=f_{\Lambda} g$ вьполнена оценка $h_{f}( \pm \pi / 2)<\varepsilon$ и ввиду (4.3) вьполнена оценка

$$
\begin{aligned}
\max \left\{h_{f}(0), h(\pi)\right\} & <\frac{12 \pi(\pi+2) \Delta_{\Lambda}}{\pi / 2-\beta} \cdot \operatorname{ctg} \beta \\
& \leqslant \frac{12 \pi(\pi+2) \Delta_{\Lambda}}{\pi / 4} \cdot \frac{1}{\operatorname{tg} \beta}=\frac{48^{2}(\pi+2)^{2} \Delta_{\Lambda}^{2}}{\varepsilon}<\frac{61000 \Delta_{\Lambda}^{2}}{\varepsilon} .
\end{aligned}
$$

Если вьполнено (0.7), то по выбору (4.3) значения $\beta$ согласно заключительной части следствия 1 функцию $g$ можно выбрать с нулями только на $i \mathbb{R}$.

Теорема 1 доказана. 
ДоКАЗАТЕЛЬСТВО СЛЕДСТВИЯ 3. В силу известной связи между полнотой и единственностью [1] достаточно показать, что сдвиг индикаторной диаграммы некоторой ЦФТ $f$, обращающейся в нуль на $\Lambda$, можно поместить в области $G$. Не умаляя обшности, можем считать, что $l=\mathbb{R}_{+}$и $x y_{+}(x) \rightarrow+\infty$ при $x \rightarrow+\infty$. Выберем $C=244000 \Delta_{\Lambda}^{2}+1$. Найдется $x_{0}$ такое, что $y_{+}(x)>C / x$ при всех $x \geqslant x_{0}$. Выберем $\varepsilon>0$ так, что $2 \varepsilon x_{0}<1$. Тогда

$$
2 \varepsilon<\frac{C}{x_{0}+122000 \Delta_{\Lambda}^{2} / \varepsilon},
$$

т.е. прямоугольник $\left\{z \in C: 0<\operatorname{Im} z<2 \varepsilon, x_{0} \leqslant \operatorname{Re} z \leqslant x_{1}\right\}$, где число $x_{1}$ равно знаменателю в правой части (4.4), лежит внутри области $G$. По теореме 1 существует ненулевая ЦФЭТ, обращающаяся в нуль на $\Lambda$, с индикаторной диаграммой, сдвиг которой содержится в указанном прямоугольнике.

Следствие 3 доказано.

\section{СПИСОК ЦИТИРОВАННОЙ ЛИТЕРАТУРЫ}

[1] Левин Б. Я. Распределение корней целых функций. М.: Физматгиз, 1956.

[2] Красичков-Терновский И.Ф. Инвариантные пространства аналитических функций. II. Спектральный синтез на выпуклых областях // Матем. сб. 1972. Т. 88. № 1. С. 3-30.

[3] Malliavin P., Rubel L. A. On small entire functions of exponential type with given zeros // Bull. Soc. Math. France. 1961. V. 89. № 2. P. 175-201.

[4] Хабибуллин Б. Н. О малости роста на мнимой оси целых функций экспоненциального типа с заданными нулями // Матем. заметки. 1988. Т. 43. № 5. С. 644-650.

[5] Хабибуллин Б. Н. О росте целых функций экспоненциального типа вдоль мнимой оси // Докл. АН СССР. 1988. Т. 302. № 2. С. 270-273.

[6] Хабибуллин Б. Н. О росте целых функций экспоненциального типа вдоль мнимой оси // Матем. сб. 1989. Т. 180. № 5. С. 706-719.

[7] Красичков И. Ф. Сравнение целых функций конечного порядка по распределению их корней // Матем. сб. 1966. Т. 66(112). С. 198-230.

[8] Хабибуллин Б. Н. Множества единственности в пространствах целых функций одной переменной // Изв. АН СССР. Сер. матем. 1991. Т. 55. № 5. С. 1101-1123.

[9] Hayman W. K. Subharmonic Functions. V. II. London: Acad. Press, 1989.

[10] Koosis P. Leçons sur le théorème de Beurling et Malliavin. Montréal: Les Publ. CRM, 1996.

[11] Gamelin T. W. Uniform Algebras and Jensen Measures. Cambridge: Cambridge Univ. Press, 1978.

[12] Хабибуллин Б. Н. Теорема о наименьшей мажоранте и ее применения. І. Целые и мероморффые функции // Изв. РАН. Сер. матем. 1993. Т. 57. № 1. С. 129-146.

[13] Хабибуллин Б. Н. Выметание на систему лучей и целые функции вполне регулярного роста // Изв. АН СССР. Сер. матем. 1991. Т. 55. № 1. С. 184-202.

[14] Ронкин Л.И.Введение в теорию целых функций многих переменных. М.: Наука, 1971.

[15] Хейман У., Кеннеди П. Субгармонические функции. М.: Мир, 1980.

Башкирский государственный университет, г. Уф̆а

Поступило E-mail: algeom@bsu.bashedu.ru

02.11.1999

Исправленный вариант 09.08.2000 